\section{Psoriasis: mit Hemmung der Tropomyosin-Rezeptor-Kinase gegen Pruritus}

\author{
Durch Hemmung der Tropomyosin-Rezeptor-Kinase A (TrkA) lässt sich bei \\ Psoriasispatienten der Juckreiz wirkungsvoll lindern. Dies geht aus einer \\ randomisierten Doppelblindstudie hervor, in der erstmals der Kinasehemmer \\ CT327 topisch verabreicht wurde.
}

$A^{\prime}$ $\mathrm{n}$ der Phase-2b-Studie nahmen 160 Patienten in vier gleich großen Gruppen teil, bei denen eine leichte bis moderat ausgeprägte Psoriasis diagnostiziert worden war, die $10 \%$ der Körperoberfläche erfasst hatte. Der Kinasehemmer CT327 wurde mithilfe einer vaselinhaltigen Salbe zweimal am Tag in den Dosierungen $0,05 \%, 0,1 \%$ oder $0,5 \%$ aufgetragen, in der Kontrollgruppe ausschließlich die Salbe. Die Schwere der Psoriasis wurde mithilfe des IGA-Scores (Investigator's Global Assessment) und des mPASI (modifizierter Psoriasis Area and Severity Index) ermittelt und der Juckreiz anhand der visuellen Analogskala (VAS) beurteilt. Behandelt wurde acht Wochen lang, wobei Gesicht und Kopfhaut ausgespart wurden. $84 \%$ der Studienteilnehmer absolvierten alle vorgesehenen Behandlungen.

Zwischen Verum- und Kontrollgruppe ergab sich bei allen Konzentrationen des Wirkstoffs kein signifikanter Unterschied beim Paramater Kontrollrate, also bei der Quote der Patienten mit Teilansprechen, vollständigem Ansprechen oder Stabilisierung der Erkrankung. Beim Parameter mPASI gab es hingegen einen statistisch signifikanten Unterschied, aber nur bei den Patienten, die die $0,05 \%$ ige Salbe auf die Hautläsionen aufgetragen hatten. Nach acht Wochen Therapie wurde in der Verumgruppe eine mPASI-Reduktion zwischen $37,1 \%$ und $42,8 \%$ erzielt, in der Kontrollgruppe hingegen nur um knapp $30 \%$.

Deutlicher war der Effekt der Behandlung mit dem gut verträglichen Kinasehemmer auf den Pruritus, dessen Ausgangswert zu Studienbeginn bei mindestens $40 \mathrm{~mm}$ von maximal $100 \mathrm{~mm}$ (stärkster Juckreiz) auf der visuellen Analogskala lag $(\mathrm{n}=108)$. Nach acht Wochen Behandlung sank der VAS-
Wert am stärksten bei Patienten, die eine Salbe mit der niedrigsten Hemmstoffkonzentration von $0,05 \%$ aufgetragen hatten, nämlich um 37,1 mm (95\%-Konfidenzintervall zwischen $-37,5$ und $6,2 ; \mathrm{p}=0,0067)$.

Die Entwicklung des TrkA-Hemmers beruht auf der Tatsache, dass die lokale Zunahme der Konzentration an Nervenwachstumsfaktor (NGF, „nerve growth factor") in der Epidermis in der Pathogenese der Psoriasis inklusive Juckreiz von Bedeutung ist. Und da der Faktor unter anderem an den TrkA-Rezeptor bindet, lässt sich die Wirkung des NGF durch einen Hemmstoff des Rezeptors beeinflussen. Beide sind an der Entstehung von Juckreiz über das TRPV1(,transient receptor potential cation channel subfamily $\mathrm{V}$ member 1")-Protein beteiligt. Es handelt sich um einen Capsaicin-Rezeptor, der auch durch endogene Agonisten und Hitze aktiviert wird. Forscher halten den Eingriff in die Signalkaskade NFG-TrkaATRPV1 zur Juckreiz-, aber auch Schmerzbekämpfung für vielversprechend.

Fazit: Mit dem TrkA-Hemmer CT327 lässt sich der Juckreiz bei Psoriasispatienten erfolgreich lindern und in einer Dosierung von $0,05 \% \mathrm{w} / \mathrm{w}$ eine signifikante Verbesserung des mPASI-Wertes erzielen. Eine Besserung des gröberen IGA-Scores, der primäre Endpunkt, wurde jedoch in der Studie nach acht Wochen Therapie nicht erzielt.

Peter Leiner

Roblin D et al. Topical TrkA Kinase Inhibitor CT327 is an Effective, Novel Therapy for the Treatment of Pruritus due to Psoriasis: Results from Experimental Studies, and Efficacy and Safety of CT327 in a Phase $2 b$ Clinical Trial in Patients with Psoriasis. Acta Derm Venereol 2015; 95: 542-8 